CAHIERS DE

NARRATOLOGIE

\section{Cahiers de Narratologie}

Analyse et théorie narratives

25 | 2013

Humour et modernité dans les littératures de langues romanes du XIXe au XXIe siècle

\title{
La contestation par l'humour. Étude contrastive de l'humour dans la littérature espagnole et française contemporaine
}

\section{María Dolores Vivero García}

\section{OpenEdition}

\section{Journals}

Édition électronique

URL : http://journals.openedition.org/narratologie/6788

DOI : 10.4000/narratologie.6788

ISSN : 1765-307X

Éditeur

LIRCES

Référence électronique

María Dolores Vivero García, « La contestation par l'humour. Étude contrastive de l'humour dans la littérature espagnole et française contemporaine », Cahiers de Narratologie [En ligne], 25 | 2013, mis en ligne le 20 décembre 2013, consulté le 19 avril 2019. URL : http://journals.openedition.org/ narratologie/6788; DOI : 10.4000/narratologie.6788

Ce document a été généré automatiquement le 19 avril 2019

Article L.111-1 du Code de la propriété intellectuelle. 


\title{
La contestation par l'humour. Étude contrastive de l'humour dans la littérature espagnole et française contemporaine
}

\author{
María Dolores Vivero García
}
[...] et je n'oublierai jamais ton rire quand perché là-haut sur la tour magnifique oiseau de l'humour tu clignais de l'œil en désignant de l'aile

les croassants oiseaux de la morale ${ }^{1}$.

1 L'humour, par son pouvoir de mettre en question les valeurs doxales, peut être une arme de combat efficace dans les littératures qui résistent aux logiques dominantes. Ses manifestations varient cependant selon les cultures, les formes de contestation par l'humour empruntant souvent des chemins diversifiés. Je me propose, dans cet article, de comparer l'humour de quelques auteurs contemporains parmi les plus représentatifs en France et en Espagne, en essayant de dégager chez ces auteurs des ressemblances et des différences en ce qui concerne les procédés utilisés, les effets de connivence suscités (essentiellement ludiques ou plus directement critiques) et les principales cibles visées par leur humour. Mais, tout d'abord, il convient de présenter brièvement le cadre méthodologique adopté. Cette étude se situe dans le cadre du projet de recherche (FFI2012-33068) financé par le Ministerio de Economía y Competitividad.

\section{Cadre méthodologique}

2 La notion d'humour semble se dérober à toute description ${ }^{2}$. Depuis des siècles, on a cherché à cerner l'essence de l'humour à partir d'études qui, malgré leur intérêt 
indéniable, n'ont pas permis de saisir ce qui caractérise en propre ce phénomène. La première difficulté vient sans doute des approches théoriques restreintes qui, comme le souligne Llera (2004), répondent à un moment historique ou à l'esthétique personnelle d'un auteur. On constate également des difficultés quand il s'agit de définir un humour national : même si des pistes importantes ont été apportées, la tendance est à réduire sa spécificité à un trait dominant - l'absurde dans l'humour anglo-saxon, l'autodérision dans l'humour juif (Stora-Sandor, 1984) -, alors que ce qui est spécifique en général c'est la combinaison de différents paramètres (comme les procédés et les effets de connivence) qui, dans ces études, ne sont pas toujours différenciés. Enfin, en ce qui concerne les procédés, malgré le grand nombre d'études dans ce domaine, il n'y a pas de catégories stables généralement admises, si bien qu'on est sans cesse confronté à la prolifération de termes (moquerie, ironie, dérision, raillerie, grotesque...), qui coexistent parfois dans une même étude, utilisés la plupart du temps dans leur sens courant, sans avoir fait l'objet d'une définition préalable. Si on veut échapper à une approche purement intuitive, il faut donc commencer par se doter de catégories rigoureusement définies en établissant des frontières conceptuelles à la base.

Dans le groupe de recherche franco-espagnol que j'anime, nous partons du constat que l'humour est un phénomène protéiforme et nous faisons l'hypothèse que l'on peut décrire chacune de ses formes comme le résultat de l'association d'un effet de connivence à un procédé ou à l'articulation de plusieurs procédés élémentaires. Pour l'établissement des catégories, notamment celles qui concernent les procédés, l'approche contrastive adoptée s'est révélée très féconde. J'en donnerai un seul exemple à propos de la tradition, dans la critique française, de séparer l'humour et l'ironie. Cette distinction bergsonienne (Bergson 1900), reprise et reformulée par Gérard Genette (2002), qui les différentie en fonction de leur caractère polémique ou non (l'ironie, au contraire de l'humour, serait toujours polémique), est reprise également, en linguistique, par Oswald Ducrot (1984), pour qui l'humour est une sorte d'ironie qui ne prend personne à partie ${ }^{3}$. Pourtant, cette dichotomie ne va pas sans poser un certain nombre de problèmes, dès qu'on est confronté à des formes d'humour espagnol souvent très critiques, comme l'hyperbole caricaturale du négatif, qui relève d'une attitude énonciative opposée à l'ironique. On se rend compte ainsi qu'inclure l'effet de connivence critique dans la définition de l'ironie peut donner lieu à des amalgames, dont le plus considérable est d'indifférencier sous cette notion des procédés très différents, au motif qu'ils suscitent la critique ou la polémique. C'est pourquoi nous séparons, dans notre cadre méthodologique, les effets de connivence suscités des procédés, et nous considérons l'humour comme une notion générique à l'intérieur de laquelle se situent différentes catégories de procédés, comme l'ironie, l'hyperbole du négatif ou la parodie, entre autres, qui peuvent se combiner entre eux et être utilisés dans différentes visées, proposant au lecteur une forme de connivence plus ou moins ludique ou plus ou moins foncièrement critique.

4 Avant de présenter très brièvement ces catégories, j'avancerai une description énonciative de l'acte humoristique, que l'on peut considérer comme un acte de langage caractérisé par une locution distanciée vis-à-vis des assertions contenues dans les énoncés ou vis-à-vis des appréciations évaluatives, dans un contexte qui à la fois légitime l'humour et permet au destinataire, en fonction de l'image qu'il a du sujet énonciateur, de prêter à celui-ci l'intention de parler de façon non sérieuse pour susciter le rire ou l'amusement. 
Dans le discours littéraire, dont la plupart des genres admettent des effets d'humour, le lecteur peut attribuer cette volonté à un personnage-locuteur qui n'adhère pas sérieusement à ce qu'il dit. Mais il n'est pas toujours aisé de savoir si les personnages se distancient humoristiquement de leur dire; on peut alors prêter cette intention à un narrateur figure d'auteur ou même à l'auteur implicite, en tant qu'hypothèse de lecture (ou image que se fait le lecteur de l'auteur à partir du texte). Par exemple, dans les romans policiers de Fred Vargas, les effets d'humour sont à mettre sur le compte de l'auteur implicite, plutôt que sur celui de certains personnages qui, comme le commissaire Adamsberg, sont des êtres "hors normes » ne faisant pas spécialement preuve d'humour et pouvant assumer sérieusement des propos qui s'écartent des modèles prévisibles.

6 Enfin, on le sait, le rire est subjectif; tout dépend en dernier lieu de l'état d'esprit du lecteur, de sa capacité à rire de quelque chose et de sa sensibilité à certaines formes d'humour.

7 C'est dans la dynamique de cet acte de langage que l'on peut situer les différentes formes de connivence recherchées et/ou suscitées, critique, ludique ou cynique, attribuées par le lecteur au personnage ou à la figure de l'auteur, en fonction de sa lecture du texte et de sa connaissance du contexte. C'est également dans le cadre de cette dynamique que l'on peut étudier les traits intrinsèques de l'humour, selon deux sortes de procédés: les énonciatifs et les sémantico-référentiels. Je viens de faire allusion aux premiers ; l'étude contrastive de l'humour français et espagnol montre, comme je le disais, l'intérêt de distinguer, outre la parodie, deux postures énonciatives contraires : d'une part, l'hyperbole caricaturale du négatif ( «sarcasme » ou « raillerie » selon les termes de Charaudeau), d'un emploi très généralisé dans le contexte espagnol, où elle sert à critiquer directement différents aspects de la vie sociale ou politique et, d'autre part, l'ironie, plus fréquente dans le contexte français, où elle consiste en général à traiter en termes apparemment valorisants une réalité qu'il s'agit de dévaloriser.

8 Ironie et exagération du négatif instaurent une distanciation ludique entre le locuteur, qui apparaît comme responsable de l'énoncé, et la position qu'exprime cet énoncé, par rapport à laquelle le locuteur donne à entendre qu'il se désolidarise. Quant à la parodie, qui est un procédé très productif dans les deux contextes, elle repose de manière spécifique sur un effet d'écho laissant voir sous le texte parodique un texte parodié reconnaissable à travers sa déformation.

9 Ces trois procédés énonciatifs peuvent s'articuler à trois autres procédés, qui portent non plus sur la distance énonciative, mais sur la manière de représenter le monde, selon notamment trois types d'incohérence : la paradoxale, qui joue avec les contradictions, l'insolite, qui rapproche des univers différents sur la base d'un aspect commun et la loufoque (ou absurde), qui associe des choses qui n'ont rien en commun entre elles.

Une fois qu'on a décrit ces six catégories de procédés élémentaires, on peut analyser leurs combinaisons, selon des relations de coordination ou de subordination, et la façon dont, suivant les contextes, elles apparaissent associées à un effet de connivence critique, ludique ou cynique.

11 J'en donnerai pour illustration l'analyse de cet énoncé de Balzac : « Il avait fait faire dans l'intérêt de la ville d'excellents chemins qui menaient à ses propriétés » (Balzac, III, 1031), cité par Ruth Amossy (2003 : 144) comme exemple d'ironie à propos de monsieur Grandet, maire de Saumur, qui ne regrette pas de quitter les honneurs municipaux. Effectivement, 
l'attitude énonciative est ironique, puisque l'évaluation positive qui se dégage de la première partie de l'énoncé «Il avait fait faire dans l'intérêt de la ville » se révèle être le support d'un blâme dès qu'on comprend que s'il a fait faire ces " excellents chemins " c'est dans son intérêt personnel. Pourtant, quand on regarde de près cet exemple, on se rend compte que, subordonné à cette énonciation ironique, la dénonçant en même temps, un paradoxe est à la base de l'effet d'humour: il nait de l'opposition entre la notion d'utilité publique et celle d'intérêt propre, perceptible dans «menaient à ses propriétés ». Si l'on tient compte, en outre, de la visée critique de cet énoncé (attribuée au narrateur balzacien), on peut conclure qu'il s'agit d'un paradoxe ironique à effet de connivence critique.

12 Le paradoxe n'étant pas nécessairement subordonné à l'ironie, on le trouvera, chez des auteurs français, mais surtout chez les espagnols, comme nous le verrons plus loin, au service de la caricature du négatif.

13 Pour de plus amples développements du cadre méthodologique, je renvoie aux travaux de Charaudeau (2006, 2011 et 2013), Charaudeau et Vivero García (à paraitre), Houdebine et Vivero García (2011) et Vivero García (2006, 2011a, 2011b et 2013).

\section{Formes d'humour}

14 J'examinerai maintenant quelques formes d'humour parmi les plus fréquentes. Tout d'abord, j'étudierai le fonctionnement de la parodie et de l'insolite caricaturaux, c'est-àdire au service de l'hyperbole caricaturale du négatif. Ces deux formes espagnoles d'humour par excellence apparaissent parfois associées, comme nous le verrons, au paradoxe caricatural. Je m'arrêterai, ensuite, sur le rôle de l'insolite non caricatural chez le poète espagnol Angel González, que je comparerai à Prévert.

\section{La parodie caricaturale}

Cette forme d'humour accentue et concentre les traits d'un discours pour le tourner en dérision. On la trouve, par exemple, dans l'extrait suivant du Prix de Montalbán, qui mime les lieux communs éculés de l'arsenal de la critique littéraire, contribuant ainsi à la caricature féroce du monde de la culture officielle :

[...] aquella eterna promesa que desde hacía diez años recibía siempre la misma crítica, del mismo crítico, en el mismo periódico: «Uno de los fenómenos más tipificables de la Nueva Narrativa Hispánica es el de Sagalés, escritor ensimismado que sólo permite proximidades a los espíritus más dispuestos a sorprenderse todavía con una literatura opuesta a las leyes del mercado, capaces de entender la lucha casi en solitario de un escritor dotado del don de la ironía secreta como instrumento de conocimiento de un universo que él solo sabe ver ${ }^{4} . .$. ».

Chez des écrivaines espagnoles, comme Rosa Montero ou Paloma Díaz-Mas, la parodie caricaturale s'en prend souvent à une façon masculine de concevoir le monde et de se représenter les femmes ou les rapports homme-femme. Dans Amado amo de Rosa Montero, c'est en effet par la parodie que passe la caricature de la mentalité des cadres, de leur machisme, de leur énervement vis-à-vis des féministes, qui ne partagent avec eux ni les valeurs de la virilité ni la culture d'entreprise dominée par la concurrence, les jalousies, les trahisons, la peur des délations, les hypocrisies et les humiliations. Parallèlement, la critique du modèle phallocrate associé à une mentalité guerrière 
emprunte, dans El rapto del Santo Grial de Paloma Díaz-Mas, les chemins de l'exagération caricaturale du discours parodié, qui est ici celui des romans de chevalerie.

\section{L'insolite caricatural}

Les rapprochements insolites sont souvent au service de l'exagération caricaturale des défauts de la cible, selon la forme d'humour la plus répandue dans le contexte espagnol: l'insolite caricatural. Ainsi en va-t-il de cette caricature de l'agressivité des chauffeurs de taxi à la sortie d'une gare en Inde :

Consiguieron optar con vida a un taxi a través de un pasillo de conductores que trataba de retenerlos, de separarlos, de trocearlos para multiplicarlos en clientes potenciales 5 .

Cette représentation insolite des chauffeurs voulant soi-disant démultiplier les clients en découpant les deux protagonistes de l'histoire (les célèbres Carvalho et Biscuter) prend une valeur intensive. Elle permet d'intensifier l'image hyperbolique de l'attitude des chauffeurs vis-à-vis des touristes et de la concurrence entre eux pour capter des clients.

Associé à des effets de connivence plus nettement critique que dans l'exemple précédent, l'insolite caricatural dévalorise chez Manuel Vázquez Montalbán l'église catholique, les socialistes ou la politique parlementaire :

[...] un tipo bajito que bebía una bebida larga con mucho hielo en el vaso y mucha melancolía en los ojos.

- ¿Alcohólico anónimo?

- No. Diputado en el Parlamento de Cataluña.

Le contestó el solitario bebedor.

$-i$ Un trago largo entre dos sesiones?

- No. Me he perdido

Un diputado perdido en las Ramblas, melancólicamente meditabundo ante un trago

largo sólo podía ser socialista.

- ¿Es usted socialista?

- ¿Se me nota ${ }^{6}$ ?

Le passage montre bien la valeur intensive que prennent les rapprochements insolites dans un contexte caricatural. En effet, le rapprochement du parlementaire aux alcooliques anonymes fonctionne au service de la caricaturale des socialistes dont l'échec des illusions politiques est exagéré hyperboliquement.

Cette même forme d'articulation de procédés joue un rôle essentiel chez un autre catalan, Eduardo Mendoza, dont l'humour destitue le pouvoir économique (lorsque, par exemple, le président d'un conseil d'administration est assimilé à un grille-pain) ou le pouvoir politique (quand l'insolite joue sur les frontières entre les ministres et les brigands). L'insolite caricatural domine par ailleurs globalement les romans policiers de cet auteur, dont le protagoniste, un détective au discours plein de bon sens, est un fou interné dans un hôpital psychiatrique, auquel a recours officieusement le commissaire Florez pour résoudre les enquêtes à sa place. Ce rufián sale, malodorant, avec des problèmes de rétention urinaire, se promenant parfois dans la rue à moitié nu, s'associe à d'autres personnages non moins insolites, le but étant de dénoncer par un humour acerbe la corruption politique.

Plus complexe habituellement que chez Mendoza, l'insolite s'associe fréquemment chez Vázquez Montalván au paradoxe, qui invite à percer à jour des opinions dominantes, dans une entreprise généralisée de dévaluation du pouvoir. Comme l'insolite, le paradoxe est 
subordonné à l'hyperbole caricaturale. L'exemple suivant cible en tout premier lieu les anciens révolutionnaires reconvertis :

La Oficina Olímpica, Preolímpica, Transolímpica, Postolímpica empleaba a las gentes en otro tiempo menos olímpicas de este mundo, gente que había hecho un viaje [...] : del marxismo leninismo a la gestión democrática institucional y finalmente a preparar todos los Olimpos que la democracia española tendría en 1992 : el Quinto Centenario del Descubrimiento de América, la Feria Internacional de Sevilla, las Olimpiadas, Madrid capital cultural de Europa. Quien no ha perdido siquiera media hora de su vida preparando la revolución, jamás sabrá qué se siente cuando años después te descubres a ti mismo prefabricando olimpos y podiums triunfales para los atletas del deporte, del comercio y de la industria. De Sierra Maestra o Olimpia. De la larga marcha a los cincuenta kilómetros marcha. De atravesar fronteras clandestinamente a negociar con los representantes de todos los fabricantes de cacao en polvo del mundo, ávidos de conseguir la concesión olímpica. De la colección completa de arrepentidos de Sierra Maesstra y de las largas marchas, escogió otra vez al "coronel Parra ", en otro tiempo autor de un manual del torturado elaborado a partir de su propia experiencia y ahora reciclado al cargo de selector de sponsors olímpicos ${ }^{7}$.

On observe comment les rapprochements insolites s'associent au jeu sur les oppositions entre les idéaux du marxisme-léninisme et la gestion institutionnelle, entre la préparation de la révolution et la fabrication des « podiums triomphaux pour les athlètes [...] du commerce et de l'industrie », entre la « Longue Marche » et autres actions liées à la révolution et celles plus juteuses liées aux affaires.

Le paradoxe, l'insolite et même la parodie, comme nous l'avons montré en 2.1., sont dominés par l'hyperbole caricaturale du négatif dans l'humour mordant de Vázquez Montalbán, qui prend pour cible toute manifestation d'une culture officielle destinée à masquer le pouvoir de l'argent et l'ambition personnelle; il critique la bourgeoisie, son esprit de classe et ses valeurs, l'esprit de consommation, le profit à outrance et la concurrence, les marchés, la finance, l'aristocratie, l'esprit militaire ${ }^{8}$. Dans son roman intitulé Les Thermes, la critique féroce du militarisme rejoint celle des principes hygiénistes (régimes, exercice physique) comme organisateurs de la vie: on peut citer la scène insolite de la prise militaire de la cuisine de l'établissement thermal par un groupe de clients au régime, dont un colonel ; celui-ci dirige les opérations et fait prisonnier le responsable de la cuisine, un homme du village qui entre inopinément; l'assimilation de la situation à une véritable opération de guerre, le langage militaire utilisé et surtout le fiasco après avoir trouvé une cuisine vide et s'être fait surprendre par un vigile, tout cela dévalue par dérision les valeurs militaires.

En somme, dans ces contextes dominés par l'exagération du négatif, les incohérences insolites sont résorbées par l'interprétation hyperbolique, non sans avoir préalablement destitué les institutions et les valeurs doxales.

\section{L'insolite non caricatural}

Cette troisième forme d'humour est très fréquente dans la littérature française. Utilisée par Fred Vargas dans une visée plutôt ludique, elle contribue de manière efficace à rompre les schémas et à renverser des opinions socialement dominantes. N'étant pas associé à un quelconque regard évaluateur, l'insolite vargassien se révèle, en effet, d'une redoutable efficacité pour bousculer des stéréotypes et pour créer des personnages masculins et féminins qui s'éloignent des conventions9. 
27 En Espagne, c'est surtout dans la poésie que l'on trouve l'insolite non caricatural. Dans sa forme la plus simple, il surgit, sous la plume du poète Ángel González, des énumérations hétéroclites comme la suivante :

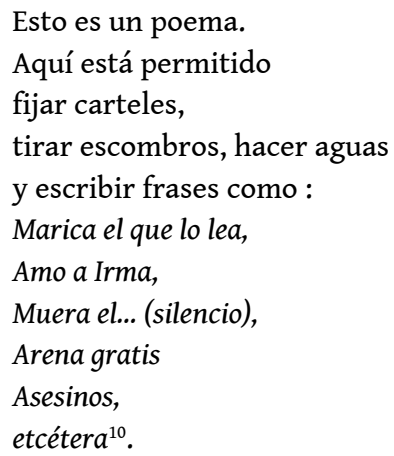

Le procédé n'est pas sans rappeler les inventaires de Prévert. Les deux poètes utilisent en effet les connexions insolites pour dévaluer et pour destituer différentes formes d'autorité, en connectant les domaines humain et animal :

J'ai mis mon képi dans la cage

et je suis sorti avec l'oiseau sur la tête

Alors

on ne salue plus

a demandé le commandant

Non

on ne salue plus

a répondu l'oiseau ${ }^{11}$;

ou bien l'animé et le non animé :

et seize et seize qu'est-ce qu'ils font?

Ils ne font rien seize et seize

et surtout pas trente-deux

de toute façon

et ils s'en vont ${ }^{12}$;

ou bien encore le divin et l'humain :

VOUS ALLEZ VOIR CE QUE VOUS ALLEZ VOIR

Une fille nue nage dans la mer

Un homme barbu marche sur l'eau

où est la merveille des merveilles

Le miracle annoncé plus haut ${ }^{13}$ ? qu'elle soit :

Vers la fin d'un discours extrêmement important

le grand homme d'État trébuchant

sur une belle phrase creuse

tombe dedans ${ }^{14}$. prendre l'adjectif « creux » au sens figuré puis au sens propre. Loin de tout grossissement caricatural, les appréciations positives « important » et «belle » dénoncent une posture énonciative ironique.

Lestorite religieuse est particulièrement visée chez les deux poètes. Dans son poème intitulé "Parque con zoológico », Angel González désacralise le discours biblique sur le mythe de la création universelle en rapprochant le paradis originel d'un parc municipal : 
PARQUE CON ZOOLÓGICO[...]

hay como una parodia del humano genuino

en su versión original, antes

de que incurriese en pena de destierro

por indebida apropiación de fruta.

"Prohibido coger flores"

¿No es casi igual

que entonces -tal como nos lo cuentan?

[...] También descansa todo,

aquí. Acuden los pañuelos

con frecuencia

a enjugar el sudor que brota de las frentes,

pero esa mancha húmeda [...]

no surge del esfuerzo necesario

para ganar el pan [...]

Vegetación y ocio, cachorros

de cocodrilo y de contribuyente :

he ahí la Creación

municipal ${ }^{15}$.

L'expression "Création municipale », parodie de "Création universelle», est dans ce poème au service de la connexion insolite visant à dévaluer le domaine religieux. De plus, dans l'avant-dernier vers, le rapprochement, au moyen d'une structure syntaxique de coordination, entre les crocodiles et les contribuables prolonge les effets d'insolite.

Prévert va jusqu'à animaliser la divinité :

Dieu est un grand lapin

il habite plus haut que la terre

tout en haut là-haut dans les cieux

dans son grand terrier nuageux.

[...]

une fois il a eu un fils

un joyeux lapin

et il l'a envoyé sur la terre

pour sauver les lapins d'en bas

et son fils a été rapidement liquidé

et on l'a appelé civet. [ragout de lapin]

Évidemment il a passé de bien mauvais moments

[...]

Dieu est aussi une grosse dinde de Noël

qui se fait manger par les riches

pour souhaiter la fête à son fils ${ }^{16}$.

De manière analogue, González compare le Christ sur la croix à un banderillero blessé par un coup de corne et transforme Dieu en taureau meurtrier :

El Cristo de Velázquez

Banderillero desganado.

Las guedejas del sueño cubren tu ojo derecho.

te quedaste dormido con los brazos alzados,

y un derrote de dios te ha atravesado el pecho.

Un piadoso pincel lavó con leves

algodones de luz tu carne herida,

y otra vez la apariencia de la vida

a florecer sobre tu piel se atreve.

No burlaste a la muerte. No pudiste.

El cuerno y el pincel, confabulados,

dejaron tu derrota confirmada. 
Fue una aventura absurda, bella y triste, que aún estremece a los aficionados:

¡qué cornada, Dios mío, qué cornada ${ }^{17}$ !

Le contraste est frappant entre la métaphore filée, qui bien qu'insolite peut être interprétée jusqu'à l'avant-dernier vers dans un registre poétique sérieux, et le registre humoristique dans lequel bascule le poème au dernier vers : en effet, l'exclamation « quel coup de corne, mon Dieu, quel coup de corne !» introduit un changement de ton et laisse apparaitre la subjectivité d'un énonciateur feignant humoristiquement d'endosser le point de vue d'un aficionado devant le spectacle d'un véritable coup de corne. La lecture glisse ainsi dans l'isotopie de la corrida, qui cesse d'être un isotopie comparante dans un contexte poétique pour devenir l'isotopie principale dans cet énoncé humoristique. À cela s'ajoute, bien sûr, le jeu issu du défigement de l'exclamation « Dios mío ", remotivée dans l'isotopie religieuse.

On retrouve, dans cet autre poème de González, ce même mécanisme de défigement et de remotivation comique, à propos de l'expression lexicalisée «Ni Dios", qui relève également de l'isotopie religieuse, le tout étant dominé par l'humanisation insolite de dieu :

ESO LO EXPLICA TODO

Ni Dios es capaz de hacer el Universo en una semana.

No descansó el septimo día.

$\mathrm{Al}$ séptimo día se cansó ${ }^{18}$.

L'insolite animalise et dévalue les intellectuels dans l'extrait suivant :

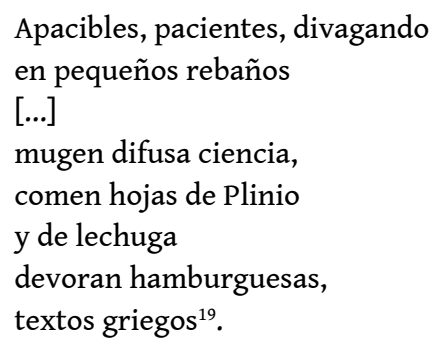

Le zeugme sémantique, que l'on aura reconnu dans les deux derniers vers, donne lieu à des rapprochements insolites chez les deux poètes: "Napoléon prit du ventre et beaucoup de pays» (J. Prévert, Paroles, p. 178), «militares [...] con artritismo y condecoraciones » («Des militaires avec de l'arthrite et des décorations $\left.{ }^{20} »\right)$ ou encore dans ces vers visant à destituer les chefs de famille :

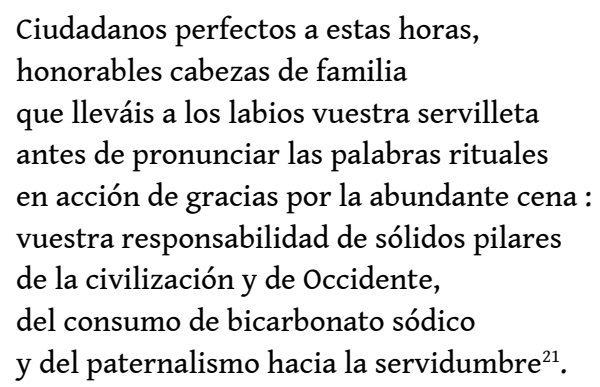

Le rapprochement des notions telles la civilisation, l'occident ou le paternalisme au "bicarbonate de soude", sans être loufoque (puisque celui-ci renvoie aux soupers abondants), est cependant insolite et destituant. Il confirme, s'il en était besoin, l'interprétation ironique des adjectifs « parfaits » et « honorables ». 
41

$$
\begin{aligned}
& \text { Quand on le laisse seul } \\
& \text { Le monde mental } \\
& \text { Ment } \\
& \text { Monumentalement }{ }^{22} \text {. } \\
& \text { Ceux qui tricolorent } \\
& \text { Ceux qui inaugurent } \\
& \text { Ceux qui croient } \\
& \text { Ceux qui croient croire } \\
& \text { Ceux qui croa-croa } \\
& \text { Crs. }
\end{aligned}
$$

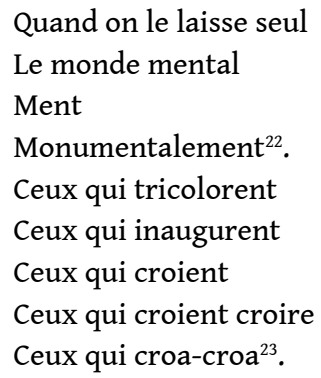
les jeux sur les ressemblances phoniques, qui abondent dans la poésie de Prévert :

Le calembour ludique donne même lieu chez le poète français à des associations nettement loufoques :

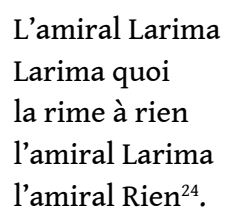

Ces associations fourmillent dans la poésie de Prévert, constamment faite de jeux de mots et de jeux de sons, bref de jeux sur le langage. Elles répondent uniquement à la logique du jeu de mots (« Un vieillard en or avec une montre en deuil (J. Prévert, Paroles, p. 227)) ou bien à une raison « textuelle » comme les structures de répétition d'un son :

Je donne des leçons de diction

Des leçons de prédication de claudication de prédiction

de malédiction de persécution de soustraction ${ }^{25}$.

la pipe au papa du pape Pie pue ${ }^{26}$.

Prônant la jouissance du verbe, Prévert destitue l'autorité de la langue et de tout ce qui est imposé par elle.

C'est là que se situe, d'après nous, la différence essentielle entre le poète français et l'Espagnol. On trouve certes dans la poésie de González des jeux de mots. J'en donnerai pour illustration le poème intitulé :

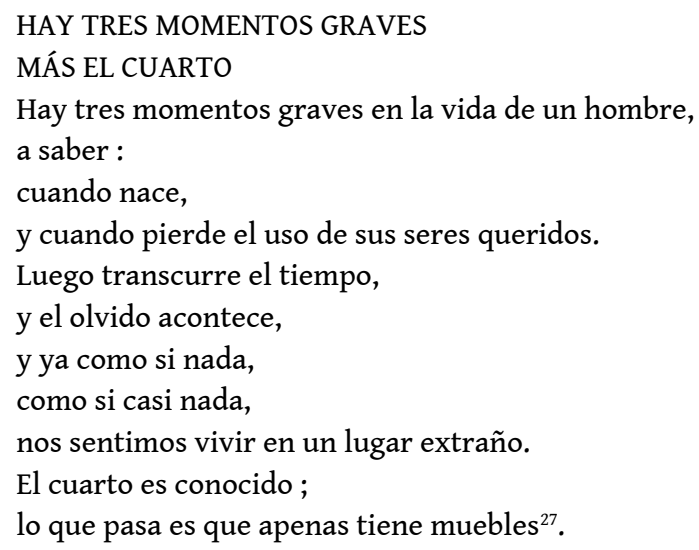

La syllepse sur «cuarto », pris au sens de "quatrième » tout d'abord, mais aussi de "chambre " (dont la nudité renvoie à la mort, ce quatrième moment dans la vie de l'homme), donne lieu à une connexion relativement loufoque. Mais, habituellement moins ludique, plus grave et plus directement cinglant que chez Prévert, l'insolite de González ne frôle pas le loufoque. Il est même parfois au service de l'hyperbole caricaturale à visée fortement critique, comme dans ce début de poème : 
Por dieciséis millones de ganglios infartados

contra catorce trillones de bacilos de Koch

ha sido elegido el singular representante

de un extenso estamento

de un harapiento estado ${ }^{28}$.

De plus, l'insolite est associé, chez González, au paradoxe, comme dans son « Introduction aux fables pour animaux ", qui ne souffre pas de citation tronquée :

Durante muchos siglos

la costumbre fue ésta :

aleccionar al hombre con historias

a cargo de animales de voz docta,

de solemne ademán o astutas tretas,

tercos en la maldad y en la codicia

o necios como el ser al que glosaban.

La humanidad les debe

parte de su virtud y su sapiencia

a asnos y leones, ratas, cuervos,

zorros, osos, cigarras y otros bichos

que sirvieron de ejemplo y moraleja,

de estímulo también y de escarmiento

en las ajenas testas animales,

al imaginativo y sutil griego,

al severo romano, al refinado

europeo,

al hombre occidental, sin ir más lejos.

Hoy quiero - y perdonad la petulancia -compensar tantos bienes recibidos

del gremio irracional

describiendo algún hecho sintomático,

algún matiz de la conducta humana

que acaso pueda ser educativo

para las aves y para los peces,

para los celentéreos y mamíferos,

dirigido lo mismo a las amebas

más simples

como a cualquier especie vertebrada.

Ya nuestra sociedad está madura,

ya el hombre dejó atrás la adolescencia

y en su vejez occidental bien puede

servir de ejemplo al perro

para que el perro sea

más perro,

y el zorro más traidor,

y el león más feroz y sanguinario,

y el asno como dicen que es el asno,

y el buey mas inhibido y menos toro.

A toda bestia que pretenda

perfeccionarse como tal

-ya sea

con fines belicistas o pacíficos,

con miras financieras o teológicas,

o por amor al arte simplemente-

no cesaré de darle este consejo :

que observe al homo sapiens, y que aprenda ${ }^{29}$.

Dans cette critique corrosive de la civilisation, on perçoit, outre l'insolite issu de l'humanisation des animaux, le jeu sur les contradictions: il y a, tout d'abord, un 
paradoxe amusant dans le fait de prétendre que les symboles de la civilisation (le subtil grecque, l'européen raffiné, l'occident) doivent leur savoir et leur vertu à des « ânes ", des « rats" ou autres «bestioles ». Ce paradoxe enchaîne sur un deuxième nourrit de l'exagération caricaturale des défauts d'une l'humanitétellement irrationnelle et accumulant tant et si bien tous les défauts attribués aux animaux, que son comportement serait désormais devenu instructif pour ceux-ci.

Enfin, il n'est pas rare, chez González, que la désacralisation passe également par la parodie du discours biblique :

PROHOMBRE

Por sus ujieres lo conoceréi ${ }^{30}$.

FINAL CONOCIDO

Después de haber comido entrambos doce nécoras, alguien dijo a Pilatos :

- ¿Y qué hacemos ahora?

El vaciló un instante y respondió

(educado, distante, indiferente) :

- Chico, tú haz lo que quieras.

Yo me lavo las manos ${ }^{31}$.

où l'on reconnaît les mots de Jésus puis ceux de Ponce Pilate.

\section{Conclusion}

51 L'humour apparait dans les deux contextes littéraires comme une arme redoutable au service de l'écriture engagée. La réussite verbale dont il fait preuve prédispose en faveur de ce qui est défendu. La comparaison de quelques auteurs français et espagnols significatifs sous cet angle nous a permis cependant de mettre en lumière quelques particularités. Il a été montré en particulier l'importance chez les romanciers espagnols de l'outrance caricaturale à effet de connivence foncièrement critique. J'en soulignerai une autre qui concerne l'emploi du loufoque. Ce procédé, qui s'inscrit dans la tradition du théâtre de l'absurde français et qui prend chez Ionesco une visée critique ${ }^{32}$, est rejeté en Espagne dès les années vingt parce qu'il est jugé, ainsi que le souligne Llera (2002: 120), comme radical et comme propre à l'humour français. On trouve certes des incongruités dans le théâtre de Miura, mais elles sont vite résorbées par l'interprétation ${ }^{33}$, si bien qu'elles ne jettent pas de doute sur la transparence du langage. Corrélativement, on trouve en général moins de jeux de mots dans la littérature espagnole que dans la française. Dans celle-ci, ils témoignent, comme chez Prévert ou encore chez Boris Vian ${ }^{34}$, d'une position de liberté face au langage (Anna Jaubert, 2011), les jeux avec les mots en général et le calembour en particulier remettant en question, outre la logique habituelle, l'autorité de la langue et sa soi-disant vocation de représenter le monde.

\section{BIBLIOGRAPHIE}

Bibliographie : 
Amossy, Ruth (2003), « Fonctions argumentatives de l'ironie balzacienne », dans Eric Bordas (éd.), Ironies balzaciennes, Saint-Cyr-sur-Loire, Christian Pirot, pp. 146-154.

Baudin, Henri (2002), « Boris Vian, paradigme d'un nouveau comique en France au milieu du XX siècle ", dans Mongi Madini (éd.), 2000 ans de rire. Permanence et modernité, Besançon, Presses universitaires franc-comtoises, pp. 213-218.

Bergson, Henri (1900), Le rire, Paris, PUF.

Charaudeau, Patrick (2006), « Des catégories pour l'humour ?», Questions de communication, 10, pp. 19-41.

- (2011), « Des catégories pour l'humour. Précisions, rectifications, compléments » dans Vivero García, María Dolores (dir.), Humour et crises sociales. Regards croisés France-Espagne, Paris, L'Harmattan, pp. 9-43.

- (2013), « De l'ironie à l'absurde et des catégories aux effets », dans Vivero García, María Dolores (dir.), Frontières de l'humour, Paris, L'Harmattan, pp. 13-26.

Charaudeau, Patrick et Vivero García, María Dolores (à paraître), « Éléments de théorie pour l'analyse discursive de l'humour ", Humoresques, 39.

Ducrot, Oswald (1984), Le dire et le dit, Paris, Minuit.

Fernandez, Manuel et Vivero García, María Dolores (2006), « L'humour dans la chronique de la presse quotidienne ", Questions de communication, 10, pp. 81-101.

Genette, Gérard (2002), « Morts de rire », dans Figures V, Paris, Seuil, pp. 134-225.

Houdebine, Anne-Marie et Vivero García, María Dolores (2011), « Quatre romancières face à la doxa. Étude de l'humour chez Paloma Díaz-Mas, Rosa Montero, Anne Garréta et Fred Vargas », dans María Dolores Vivero Garcia, Humour et crises sociales. Regards croisés France-Espagne, Paris, L'Harmattan, pp. 189-202.

Jardiel Poncela, Emilio (1929), Amor se escribe sin hache, Madrid, Cátedra.

Jaubert, Anna (2011), « Le calembour ou la pragmatique du trait /facile/ », Le français moderne, 79-1, pp. 33-43.

Llera Ruíz, José Antonio (2002), « Los procedimientos de la comicidad en el primer teatro de Miguel Mihura y de Eugène Ionesco », Exemplaria : Revista de literatura comparada, 6, pp. 97-122.

- (2004), « La investigación en torno al humor verbal », Revista de literatura, LXVI, 132, pp. 527-535.

Vivero García, María Dolores (2006), « Procedimientos discursivos y formas de humor en las columnas periodísticas francesas y españolas », Sintagma. Revista de Lingüística, 18, pp. 67-80.

- (2010), «L'humour dans l'enquête criminelle chez Fred Vargas », dans Menegaldo, Gilles et Petit, Maryse (dir.), Manières de noir. La fiction policière contemporaine, Presses Universitaires de Rennes, pp. 251-263.

- (2011a), «L'ironie, le sarcasme, l'insolite... peuvent-ils bousculer les valeurs dominantes? », dans Vivero García, María Dolores (dir.), Humour et crises sociales. Regards croisés France-Espagne, Paris, L'Harmattan, pp. 45-56.

- (2011b), « Humour, engagement et création littéraire chez Anne Garréta », Women in French Studies, 19, pp. 85-93. 
- (2013), « Catégories de procédés et formes d'humour en France et en Espagne » dans Vivero García, María Dolores (dir.), Frontières de l'humour, Paris, L'Harmattan, pp. 111-129.

\section{NOTES}

1. Jacques Prévert, Paroles, Paris, Gallimard, 1949, 219. Les pages indiquées dans les références à Paroles tout au long de l'article renvoient à cette édition.

2. Jardiel Poncela disait à ce propos : «Tratar de definir el humorismo es como pretender clavar por el ala una mariposa, utilizando de aguijón un poste del telégrafo» (1929: 93-94). Cf. bibliographie en fin d'article.

3. Selon la terminologie de Ducrot (2010), le contenu absurde qui apparaît comme conçu est finalement exclu. On s'en tiendra à l'ancienne terminologie, qui est plus connue.

4. Vázquez Montalbán, El premio, Barcelona, Planeta, 1996, pp. 17-18. « [...] l'éternelle promesse qui depuis dix ans recevait toujours la même critique, du même critique, dans le même journal : "Un des phénomènes les plus caractéristiques du Nouveau roman hispanique, c'est Sagalés, écrivain rêveur qui n'accorde sa compagnie qu'aux esprits encore prêts à s'étonner devant une littérature méprisant les lois du marché, encore capables de comprendre la lutte presque solitaire d'un écrivain dont l'ironie secrète était le seul moyen de découvrir un univers que lui seul pouvait voir..." » (Le prix, Christian Bourgois, 1999, p. 20).

5. (M. Vázquez Montalbán, Milenio Carvalho I. Rumbo a Kabul, dans El círculo virtuoso, Barcelona, Planeta, 2004, p. 530). « Encore vivants, ils réussirent à prendre un taxi après être passés par une haie de chauffeurs qui s'accrochaient à eux, essayaient de les séparer, de les couper en morceaux pour les démultiplier en clients potentiels (Milenio. Une enquête de Pepe Carvalho. Cap sur Kaboul, Christian Bourgois, 2006, p. 338).

6. Vázquez Montalbán, El laberinto griego, Barcelona, Planeta, p. 64. « [...] un type bas sur pattes qui s'imbibait méthodiquement avec beaucoup de glace dans un grand verre et la même dose de mélancolie au fond des yeux.

- Alcoolique anonyme?

— Non. Député au Parlement de Catalogne, lui répondit le buveur solitaire.

— On se rince la dalle entre deux sessions.

- Non. Je me suis perdu.

Un député mélancolique et songeur, perdu sur les Ramblas et qui se rince la dalle, ne pouvait être que socialiste.

— Vous êtes socialiste?

— Ça se voit?» (Le labyrinthe grec, Points, 2009, pp. 72-73).

7. Vázquez Montalbán, El laberinto griego, Barcelona, Planeta, p. 32-33. «Le Comité d'organisation olympique, préolympique, transolympique et postolympique employait des gens qui avaient été autrefois les moins olympiques du monde, des gens qui avaient suivi une trajectoire [...]: du marxisme-léninisme à la gestion démocratique institutionnelle, ils s'étaient attelés à la préparation des Olympes qui attendaient la démocratie espagnole en 1992: le Cinquième Centenaire de la découverte de l'Amérique, l'Exposition universelle de Séville, les jeux Olympiques, Madrid capitale culturelle de l'Europe. Qui n'a pas consacré au moins une demiheure de sa vie à préparer la révolution ne saura jamais ce qu'on peut éprouver quand, des années après, on se découvre en train de fabriquer des Olympes, des podiums triomphaux pour les athlètes du sport, du commerce et de l'industrie. De la sierra Maestra à Olympie. De la Longue Marche à la marche des cinquante kilomètres. Des franchissements clandestins des frontières aux négociations avec les représentants de tous les fabricants de cacao en poudre du monde, avides d'obtenir la concession olympique. Dans la collection complète des repentis de la sierra 
Maestra et des longues marches, il choisit de nouveau le "colonel Parra", autrefois auteur d'un manuel du torturé fondé sur sa propre expérience, aujourd'hui recyclé dans la sélection des sponsors olympiques » (Le labyrinthe grec, Points, 2009, pp. 36-37).

8. Considérant que l'humour de Vázquez Montalbán dénonce une destinée sociale impudique dominée par l'argent, García Montero, note très justement que «El Premio soporta, además de la vanidad histérica de los letraheridos y la perversión de los puros, la cultura desmedida e irresponsable del pelotazo en un tiempo que considera trasnochada toda intención ética y traiciona las ilusiones socialistas al identificar la modernidad con las privatizaciones y el glamour del dinero » (Luis García Montero, "La sociedad y la compasión », prólogo de Manuel Vázquez Montalbán, Carvalho. Puente aéreo, vol. 2, Barcelona, Planeta, 2012, p. 11).

9. Pour une étude plus développée de l'humour vargassien, je renvoie à Vivero García 2010 et Houdebine \& Vivero García 2011.

10. A. González, Poemas, Madrid, Cátedra, 1980, p. 170.

«Ceci est un poème.

Il y est permis d'y

mettre des affiches,

de jeter des décombres, d'uriner dessus

et d'écrire des phrases comme:

Celui qui lit ça est une pédale

J'aime Irma,

Mort aux... (silence),

Sable gratuit

Assassins,

et cetera ».

La poésie d'Angel González n'ayant pas été à ce jour publiée en français, toutes les traductions des poèmes sont de moi. J'ai plaisir à remercier Christine Di Benedetto de sa relecture des traductions et de ses suggestions pertinentes.

11. J. Prévert, Paroles, p. 170.

12. J. Prévert, Paroles, p. 143.

13. J. Prévert, Paroles, p. 175.

14. J. Prévert, Paroles, p. 215.

15. A. González, Palabra sobre palabra, Barcelona, Barral, 1972, pp. 203-204.

«PARC AVEC ZOO

[...]

il y a comme une parodie de l'humain authentique

dans sa version originale, avant

qu'il n'encoure le châtiment de l'exil

pour appropriation illicite de fruits.

"Interdit de cueillir des fleurs"

N'est-ce pas presque pareil

qu'alors - d'après ce qu'on nous raconte?

[...] Tout repose également,

ici. Les mouchoirs viennent

souvent

essuyer la sueur qui coule sur les fronts,

mais cette tache humide [...]

ne provient pas de l'effort nécessaire

pour gagner son pain [...]

Végétation et loisirs, petits

crocodiles et petits contribuables: 
voilà la Création

municipale ».

16. J. Prévert, Paroles, pp. 165-166.

17. A. González, Prosemas o menos, Hiperión, 1985, p. 29.

« Le CHRIST DE VÉLASQUEZ

Banderillero apathique.

Les tignasses du sommeil couvrent ton œil droit.

tu t'es endormi avec les bras levés,

et une défaite de Dieu a traversé ta poitrine.

Un pieux pinceau a lavé avec de légers

cotons de lumière ta chair blessée,

et de nouveau l'apparence de la vie

ose fleurir sur ta peau.

Tu n'as pas trompé la mort. Tu n'as pas pu.

La corne et le pinceau, confabulés,

ont confirmé ta défaite.

Ce fut une aventure absurde, belle et triste,

qui émeut encore aujourd'hui les aficionados :

quel coup de corne, mon Dieu, quel coup de corne !»

18. A. González, Prosemas o menos, Hiperión, 1985, p. 34.

«CELA EXPLIQUE TOUT

Même Dieu n'est pas capable de faire l'Univers en une semaine

Il ne s'est pas reposé le septième jour.

Le septième jour il en a eu assez ».

19. A. González, Poemas, Cátedra, 1980, p. 203.

«Paisibles, patients, divagant

en petits troupeaux

[...]

ils mugissent de la science diffuse,

ils mangent des feuilles de Pline

et de laitue

ils dévorent des hamburgers,

des textes grecs"

20. A. González, Poemas, Cátedra, 1980, p. 212.

21. A. González, Poemas, Cátedra, 1980, p. 175.

«Citoyens parfaits à cette heure-ci

honorables chefs de famille

qui portez à vos lèvres votre serviette

avant de prononcer les mots rituels

en action de grâce pour le souper abondant :

votre responsabilité de solides piliers

de la civilisation et de l'Occident

de la consommation de bicarbonate de soude

et du paternalisme envers les domestiques [...]».

22. J. Prévert, Paroles, p. 212.

23. J. Prévert, Paroles, p. 5.

24. J. Prévert, Paroles, p. 22.

25. J. Prévert, Paroles, p. 211.

26. J. Prévert, Paroles, p. 110. 
27. A. González, Prosemas o menos, Madrid, Hiperión, 1985, p. 41.

«IL Y A TROIS MOMENTS GRAVES

PLUS LE QUATRIÈME

Il y a trois moments graves dans la vie d'un homme,

à savoir :

quand il naît,

et quand il perd l'usage de ses êtres bien aimés.

Après le temps passe,

et l'oubli arrive,

et c'est déjà comme si rien,

comme si presque rien n'était arrivé,

on se sent vivre dans un lieu étrange.

Le quatrième est connu ;

Mais il a bien peu de meubles "

28. A. González, Palabra sobre palabra, Barcelona, Barral, p. 152.

«Par seize millions de ganglions infarcis

contre quatorze trillions de bacilles de Koch

a été élu le singulier représentant

d'un domaine étendu

d'un état en haillons ».

29. A. González, Palabra sobre palabra, Barcelona, Barral, p. 161.

«INTRODUCTION AUX FABLES

POUR ANIMAUX

Durant de nombreux siècles

l'usage fut celui-ci :

édifier l'homme par des histoires

d'animaux à la voix docte,

aux gestes solennels et aux ruses astucieuses,

obstinés dans le mal et la cupidité

ou abrutis comme l'être qu'ils glosaient.

L'humanité doit

une partie de sa vertu et de son savoir

aux ânes et aux lions, aux rats, aux corbeaux,

aux renards, aux ours, aux cigales et autres bestioles

qui ont servi d'exemple et de morale,

de stimulation aussi, et de leçon

pourtant infligée aux animaux,

au Grec imaginatif et subtil

au Romain sévère, au raffiné

Européen,

à l'homme occidental sans chercher plus loin.

Aujourd'hui je veux - pardon pour mon arrogance -

compenser tant de bienfaits reçus

de l'irrationnelle guilde

en décrivant quelques faits symptomatiques,

quelques nuances de la conduite humaine

qui pourraient être pédagogiques

pour les oiseaux et pour les poissons,

pour les cœlentérés et les mammifères,

adressé aussi bien aux amibes 
les plus simples

qu'à n'importe quelle espèce vertébrée.

Notre société est maintenant mûre,

l'homme est maintenant sorti de son adolescence

et dans sa vieillesse occidentale il peut bien

servir d'exemple au chien

pour que le chien soit

plus chien,

et pour que le renard soit plus traître,

et le lion plus féroce et sanguinaire,

et l'âne comme ils disent qu'est l'âne,

et le bœuf plus inhibé et moins taureau.

À toute bestiole qui prétendrait

se perfectionner en tant que telle

- que ce soit

à des fins bellicistes ou pacifistes,

avec des vues financières ou théologiques,

ou bien par amour de l'art tout simplement -

je ne cesserai de lui donner ce conseil :

qu'elle observe l'homo sapiens, et qu'elle apprenne ».

30. A. González, Palabra sobre palabra, Barcelona, Barral, p. 169.

«PROHOMME

Par ses huissiers vous le connaîtrez ».

31. A. González, Palabra sobre palabra, Barcelona, Barral, p. 278.

« FIN CONNUE

Après avoir mangé à eux deux douze crabes,

quelqu'un dit à Pilate :

- Et que fait-on maintenant?

Lui, hésita un instant et répondit

(courtois, distant, indifférent) :

- Eh bien, toi, fais ce que tu voudras.

Moi, je me lave les mains ».

32. Chez d'autres auteurs français, comme Boris Vian, il suscite de effets de connivence plus purement ludique :

«De la musique ennuyeuse comme des petits pois trop gros » (Chroniques de jazz, p. 33, cité dans

H. Baudin, 2002, p. 216).

33. Chez Miura, «la incongruencia encuentra una resolución », comme le note Llera (2002, p. 120).

34. Comme le remarque très justement $\mathrm{H}$. Baudin, chez Vian « le mot d'esprit fait place au libre jeu avec les mots, y compris les calembours et à-peu-près calamiteux ou les astuces sophistiquées: la langue et la raison voient leurs règles agressées, de l'orthographe à la rhétorique et à la logique»( H. Baudin, 2002, p. 213). Baudin donne comme exemple de protestation orthographique contestataire cette phrase de Boris Vian : c'est « inhumain, inhutile, inhgnoble et inhadmissible » (Chroniques de Jazz, p. 24, cité par Baudin p. 215). 


\section{RÉSUMÉS}

Le propos de cet article consiste à étudier, selon une approche comparative, l'humour de quelques auteurs contemporains en France et en Espagne, dans le but de dégager des ressemblances et des différences en ce qui concerne les cibles, les procédés utilisés et les effets de connivence. L'analyse, selon le cadre méthodologique présenté, se focalise sur deux formes d'humour critique dominées par l'exagération caricaturale d'une évaluation péjorative: la parodie caricaturale et l'insolite caricatural. La dernière partie de l'article est consacrée à une troisième forme d'humour, l'insolite non caricatural, tel qu'il surgit dans la poésie de Prévert et chez le poète espagnol Angel González; il y est souligné l'importance des jeux de mots dans le contexte français. Globalement, l'humour apparaît, dans les deux contextes littéraires, comme une arme au service de l'écriture engagée.

El objetivo de este artículo es estudiar, desde una perspectiva contrastiva, el humor de varios autores contemporáneos franceses y españoles para poner de manifiesto las divergencias y las similitudes en cuanto a los procedimientos utilizados, los efectos de connivencia y los blancos del humor. Tras la presentación del marco metodológico, el análisis se centra en dos formas de humor crítico dominadas por la exageración caricatural de una apreciación negativa : la parodia caricatural y el insólito caricatural, ambas frecuentes en la literatura española. La última parte del artículo está dedicada a una tercera forma de humor, el insólito no caricatural, tal y como aparece en la poesía de Jacques Prévert y en la del español Ángel González. Queda puesto de manifiesto el importante papel que juegan en el contexto francés los juegos de palabras. Globalmente, en ambos contextos literarios, el humor funciona como un arma al servicio de la escritura comprometida.

INDEX

Index géographique : France, Espagne

Index chronologique : XXe siècle

Mots-clés : humour, insolite, parodie, hyperbole, littérature contemporaine

\section{AUTEUR}

\section{MARÍA DOLORES VIVERO GARCÍA}

Universidad Autónoma de Madrid 